\title{
Healthcare utilization and costs associated with $S$. aureus and $P$. aeruginosa pneumonia in the intensive care unit: a retrospective observational cohort study in a US claims database
}

\author{
Moe H. Kyaw ${ }^{1,2}$, David M. Kern ${ }^{3}$, Siting Zhou ${ }^{3}$, Ozgur Tunceli ${ }^{3}$, Hasan S. Jafri ${ }^{1}$ and Judith Falloon ${ }^{*}$
}

\begin{abstract}
Background: Staphylococcus aureus and Pseudomonas aeruginosa are major causes of pneumonia in intensive care unit (ICU) patients. Limited data exist regarding the health economic impact of $S$. aureus and $P$. aeruginosa pneumonias in the ICU setting.

Methods: We conducted a retrospective observational cohort study using a 29.6 million enrollee US medical and pharmacy administrative claims database. ICU patients with S. aureus or P. aeruginosa infection per International Classification of Diseases, 9th ed. coding between 01/01/2007-8/31/2012 were compared with ICU patients without any pneumonia or infections of interest. Primary outcomes were costs in 2012 US dollars, healthcare utilization and all-cause mortality associated with hospital-acquired $S$. aureus or $P$. aeruginosa pneumonia, and the relative odds of incurring higher costs due to a comorbid condition.

Results: Patients with S. aureus or $P$. aeruginosa pneumonia had longer mean hospital (37.9 or 55.4 vs 7.2 days, $P<.001)$ and ICU stays (6.9 or 14.8 vs 1.1 days, $P<.001)$, a higher rate of mechanical ventilation ( $62.6 \%$ or $62.3 \%$ vs $7.4 \%, P<.001)$, higher mortality $(16.0 \%$ or $20.2 \%$ vs $3.1 \%, P<.001)$, and higher total mean hospitalization costs $(\$ 146,978$ or $\$ 213,104$ vs $\$ 33,851, P<.001)$ vs controls. Pneumonia survivors had significantly increased risk of rehospitalization within 30 days ( $27.2 \%$ or $31.1 \%$ vs $15.3 \%, P<.001)$. Comorbid conditions were not associated with increased cost in the pneumonia cohorts.
\end{abstract}

Conclusions: Healthcare costs and resource utilization were high among ICU patients with S. aureus or $P$. aeruginosa pneumonia. Reducing the incidence of these infections could lead to substantial cost savings in the United States.

Keywords: ICU, Pneumonia, Health economics, Resource utilization, S. aureus, P. aeruginosa

\section{Background}

Staphylococcus aureus (S. aureus) and Pseudomonas aeruginosa ( $P$. aeruginosa) are responsible for much of the hospital-acquired pneumonia in the United States, accounting for approximately $28 \%-47 \%$ and $18 \%$ of hospitalizations, respectively [1, 2]. Despite preventive strategies, ventilator-associated pneumonia (VAP) remains the most frequent infection occurring in ICU patients [3]. S. aureus and P. aeruginosa cause $20 \%-31 \%$

\footnotetext{
* Correspondence: falloonj@medimmune.com

'Medlmmune, Gaithersburg, USA

Full list of author information is available at the end of the article
}

and $21 \%-24 \%$ of VAP, [4-6] and $14 \%$ and $13 \%$ of non-VAP in intensive care unit (ICU) patients, respectively, in the United States [4]. These infections are associated with excess costs, lengths of stay, and mortality rates [2, 7-11]. Furthermore, infections due to antibiotic-resistant strains of $S$. aureus $[6,12,13]$ and $P$. aeruginosa [14-18] add considerable disease burden and are associated with significantly higher costs, lengths of stay, and mortality versus infections caused by antibiotic-susceptible strains.

Limited data exist regarding the pharmacoeconomic and health outcomes of $S$. aureus and $P$. aeruginosa

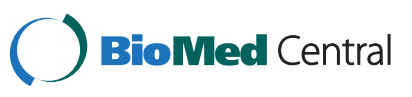


pneumonias in the ICU setting. This study assesses the impact of S. aureus or P. aeruginosa pneumonia in ICU patients on healthcare costs, utilization, and mortality both during hospitalization and subsequent to discharge from the hospital.

\section{Methods \\ Design}

This retrospective observational cohort study of administrative claims data from the HealthCore Integrated Research Environment for service dates from 01/01/2006 through 11/30/2012 was developed to assess costs and outcomes of $S$. aureus and $P$. aeruginosa pneumonia in ICU patients to guide the development of monoclonal antibodies designed to prevent these illnesses $[19,20]$. The HealthCore database contains longitudinal claims data from one of the largest commercially insured population in the United States, including approximately 29.6 million enrollees in commercial health plans at the time of this study. On average, the population included in the HealthCore database has a higher household income, a lower proportion of individuals older than 65 years, and a higher proportion of whites compared to the overall US population. The observational and longitudinal aspects of the retrospective cohort design facilitated simultaneous capture of prior and follow-up data relative to the index event. The study was conducted in compliance with US federal regulations, the Health Insurance Portability and Accountability Act, and the Helsinki Declaration. Patientspecific data was de-identified, therefore, informed consent and institutional review board or ethics committee approval were not required for this study.

\section{Study population}

The eligible population comprised patients with $\geq 1$ inpatient hospitalization that included an ICU stay and an admission and discharge between 01/01/2007 and 08/31/ 2012 (Fig. 1). Admissions to medical, surgical, cardiac,

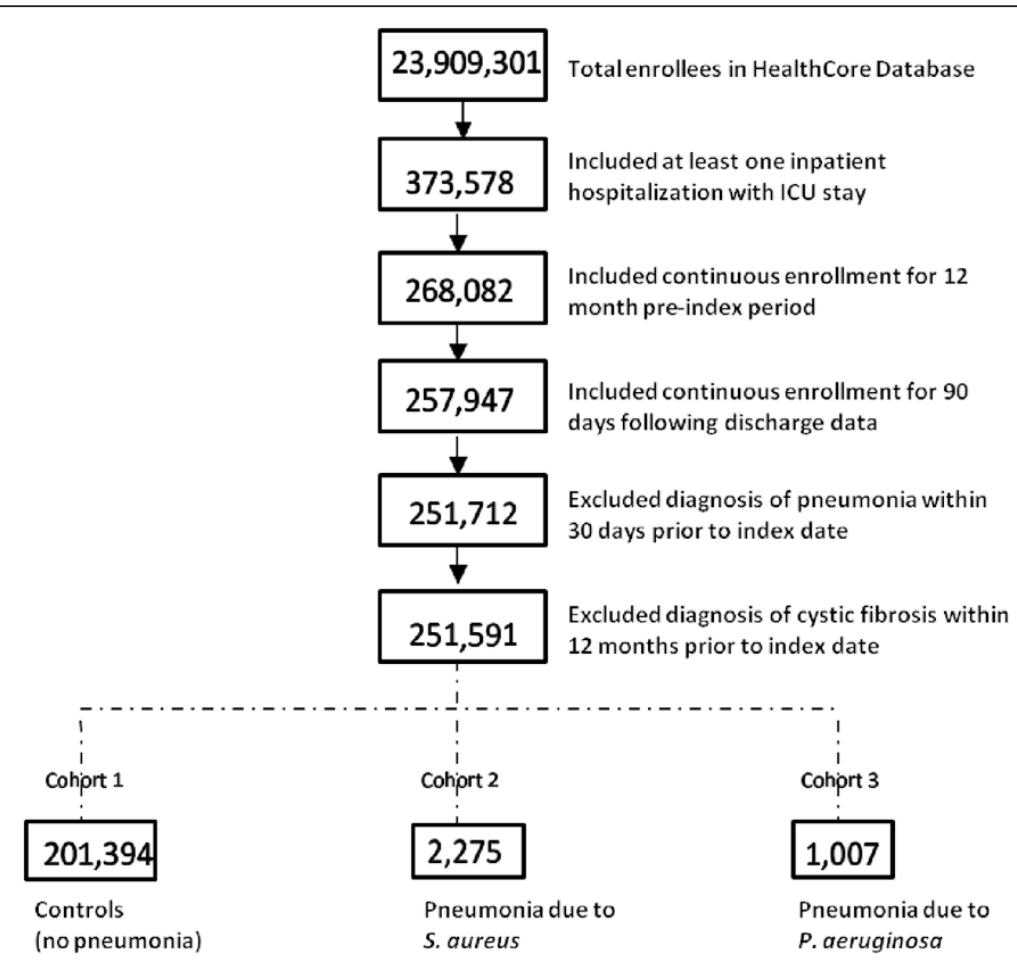

Fig. 1 Inclusion/exclusion criteria, cohort definition, and patient counts. Cohort 1: Controls (no pneumonia); excluded the following conditions and Cohort 2 and Cohort 3: Viral pneumonia (ICD-9 dx 480.xx); Other pneumonia with specified bacteria: Pneumococcal pneumonia (481.xx), Other specified bacterial pneumonia (482.0x, 482.2x, 482.3x, 482.8x), Bronchopneumonia (485.xx); Pneumonia due to unspecified bacteria: Unspecified bacterial pneumonia (482.9x), Pneumonia, organism unspecified [bacterial or viral] (486.xx), Ventilator associated pneumonia (VAP) (997.31); Other pneumonias: Aspiration Pneumonia (507.0x), Post-procedural pneumonia (997.32); Septicemia due to staphylococcus or pseudomonas: Staphylococcal septicemia, unspecified (038.10), Methicillin susceptible Staphylococcus aureus septicemia (038.11), Methicillin resistant Staphylococcus aureus septicemia (038.12), Septicemia due to pseudomonas (038.43); Other bacterial infections due to staphylococcus or pseudomonas: Staphylococcus, unspecified (041.10), Methicillin susceptible Staphylococcus aureus (041.11), Methicillin resistant Staphylococcus aureus (041.12), Pseudomonas (041.7); Staphylococcal meningitis (320.3x); Bacteremia (790.7x). Cohort 2: Pneumonia due to staphylococcus (ICD-9 diagnosis: 482.4x). Cohort 3: Pneumonia due to pseudomonas (ICD-9 diagnosis: 482.1x). Cohort 2 and Cohort 3 are not necessarily mutually exclusive. Patients with diagnosis codes for both pneumonia due to staphylococcus and pneumonia due to pseudomonas during the index hospitalization visit are included in both cohorts $(N=132)$ 
pediatric (including neonatal) ICUs were included. The first inpatient hospitalization that included any stay in an ICU was defined as the index hospitalization. To permit the identification of comorbid conditions and to assess long-term outcomes, eligible patients had $\geq 1$ year of health plan enrollment before the admission date with continuous enrollment during the inpatient episode and $\geq 90$ days of enrollment following the discharge date (unless death occurred first). Patients diagnosed with pneumonia within 30 days and patients diagnosed with cystic fibrosis within 12 months before the index period were excluded. The observation period for each patient was divided into pre-index, index, and 2 post-index time intervals. These were defined, respectively, as the 12 months before the index hospitalization, the index hospitalization itself, and both 30- and 90-day follow up periods post discharge from the index hospitalization.

Eligible ICU patients were divided into 3 primary groups based on International Classification of Disease Clinical Modification, Ninth revision (ICD-9) diagnosis codes: patients not diagnosed with pneumonia (Cohort 1-controls), patients with S. aureus pneumonia (Cohort 2), and patients with $P$. aeruginosa pneumonia (Cohort 3) (Fig. 1). Both primary and secondary diagnosis codes for the index hospitalizationwere used to assign subjects into groups.

\section{Outcomes}

The main study outcomes included the total healthcare cost of the index hospitalization, the total length of stay, all-cause mortality during the index hospitalization, and the relative odds if the presence of a comorbid condition would result in higher total index hospitalization cost. The study also examined mechanical ventilation and ICU length of stay during the index hospitalization, and all-cause healthcare costs, all-cause mortality, hospital readmission, hospital and ICU lengths of stay, and antibiotic use during the post-index periods.

A comorbid condition was defined as the presence of $\geq 1$ medical claim with a diagnosis code for the condition of interest for an individual during the 12 months prior to the index hospitalization. The total cost of care, including costs paid by the patient and those paid by the health plan, are included in all cost calculations. Post-index costs are reported as those incurred during inpatient hospitalizations (inpatient costs) as well as costs incurred in all places of service (total all-cause costs). Antibiotic use was captured via pharmacy and medical claims. The administrative claims data were linked to the Social Security Death Index to capture mortality during the post-index period.

\section{Analysis}

Statistical analyses of the $S$. aureus and P. aeruginosa cohorts were performed separately against the control cohort. Cost outcomes were analyzed using a generalized linear model with gamma distribution and a log link function. Unadjusted differences in mean costs were reported for pre- and post-index periods, while means adjusted for covariates were reported for the primary outcome of index costs. Covariates for adjusted cost models included age (continuously), gender, health plan type, geographic region, Deyo-Charlson comorbidity index score (DCI), [21] and the analogous pre-index cost for index and post-index cost analyses only (index and post-index hospitalization cost models adjusted for pre-index hospitalization costs, total all-cause post-index costs adjusted for total preindex costs).

Count variables and lengths of stay were analyzed using a generalized linear model with a negative binomial distribution with a log link function. Differences in index hospitalization length of stay were adjusted for the same covariates listed above, while unadjusted mean differences are reported for all pre- and post-index models. Logistic regression was used to analyze dichotomous variables with results reported as unadjusted odds ratios. Survival rates were calculated using Kaplan-Meier analysis, and unadjusted Cox-proportional hazards were used to analyze differences in survival between cohorts. The association between comorbid conditions and index hospitalization cost was analyzed separately within each cohort by dividing each cohort into quintiles of index hospitalization cost and calculating the prevalence of each comorbid condition within each quintile. Ordinal logistic regression was used to determine whether each comorbid condition was associated with higher cost quintiles, with results reported as unadjusted odds ratios.

For all variables, $95 \%$ confidence intervals were presented. The magnitude of point estimates and the width of confidence intervals were used primarily to interpret results. Nominal $P$-values were calculated without adjustment for multiplicity; $P<0.05$ was considered statistically significant.

\section{Results}

In total, 251,591 patients were included in the final analysis. Figure 1 shows the inclusion/exclusion criteria, definitions, and sample size of patient cohorts. Baseline demographic characteristics of patients at the time of index hospitalization are summarized in Additional file 1: Table S1. Compared with controls, pneumonia patients were more likely to be older (mean age $S$. aureus: 60.5 years; $P$. aeruginosa: 62.8 years vs controls: 57.5 years) and male (S. aureus: $59.3 \%$; P. aeruginosa: $61.5 \%$ vs controls: $56.3 \%)$. Most patients in each cohort were covered by a preferred-provider type health plan (68 \%-70 \%).

\section{Costs}

Table 1 presents a summary of the total and inpatient costs for the pre-index, index, and post-index (30 and 
Table 1 Healthcare costs for ICU patients with S. aureus or P. aeruginosa pneumonia versus controls

\begin{tabular}{|c|c|c|c|c|c|c|c|}
\hline & \multirow[b]{2}{*}{$\begin{array}{l}\text { No pneumonia, } \\
\text { mean (SD) } \\
(n=201,394)\end{array}$} & \multirow[b]{2}{*}{$\begin{array}{l}\text { S. aureus } \\
\text { pneumonia, } \\
\text { mean (SD) } \\
(n=2275)\end{array}$} & \multirow[b]{2}{*}{$\begin{array}{l}\text { P. aeruginosa } \\
\text { pneumonia, mean } \\
\text { (SD) }(n=1007)\end{array}$} & \multicolumn{2}{|c|}{ Pneumonia due to $S$. aureus vs No pneumonia } & \multicolumn{2}{|c|}{ Pneumonia due to $P$. aeruginosa vs No pneumonia } \\
\hline & & & & $\begin{array}{l}\text { Difference }^{a} \\
(95 \% \mathrm{Cl})\end{array}$ & $P$ value $^{a}$ & $\begin{array}{l}\text { Difference }^{b} \\
(95 \% \mathrm{Cl})\end{array}$ & $P$ value ${ }^{b}$ \\
\hline \multicolumn{8}{|l|}{ Pre-index costs, ${ }^{\mathrm{C}} \$$ (12 months) } \\
\hline Total all-cause costs & $17,379(38,923)$ & $24,865(55,676)$ & $29,364(65,771)$ & $7,487(6,024,9,041)$ & $<.001$ & $11,985(9,436,14,776)$ & $<.001$ \\
\hline Inpatient hospitalization costs & $5,985(25,095)$ & $10,389(40,126)$ & $11,885(47,091)$ & $4,405(3,274,5,674)$ & $<.001$ & $5,900(4,015,8,140)$ & $<.001$ \\
\hline \multicolumn{8}{|l|}{ Index hospitalization costs, ${ }^{c}$ \$ } \\
\hline Total costs & $33,851(51,770)$ & $146,978(235,598)$ & $213,104(338,552)$ & $100,216(94,346,106,360)$ & $<.001$ & $159,361(146,758,172,856)$ & $<.001$ \\
\hline \multicolumn{8}{|l|}{ Post-index costs, ${ }^{c} \$$ (30 days) } \\
\hline Total all-cause costs & $4,732(18,044)$ & $8,243(23,838)$ & $11,122(42,503)$ & $3,773(3,236,4,348)$ & $<.001$ & $6,327(5,285,7,484)$ & $<.001$ \\
\hline Inpatient hospitalization costs & $2,610(17,052)$ & $4,836(22,950)$ & $7,447(41,852)$ & $2,710(2,147,3,343)$ & $<.001$ & $5,139(3,924,6,590)$ & $<.001$ \\
\hline \multicolumn{8}{|l|}{ Post-index costs, ${ }^{c} \$$ (90 days) } \\
\hline Total all-cause costs & $10,575(27,653)$ & $17,460(39,286)$ & $22,277(54,113)$ & $7,250(6,231,8,335)$ & $<.001$ & $10,842(9,014,12,852)$ & $<.001$ \\
\hline Inpatient hospitalization costs & $4,913(23,187)$ & $9,281(35,261)$ & $13,527(50,214)$ & $4,731(3,727,5,860)$ & $<.001$ & $8118(6,110,10,513)$ & $<.001$ \\
\hline \multicolumn{8}{|c|}{ Index hospitalization + post-index costs $\left(30\right.$ days) ${ }_{1}^{c} \$$} \\
\hline Total all-cause costs & $38,583(57,402)$ & $155,221(240,527)$ & $224,226(346,331)$ & $101,579(95,681,107,742)$ & $<.001$ & $162,756(150,118,176,254)$ & $<.001$ \\
\hline Inpatient hospitalization costs & $36,461(56,270)$ & $151,813(239,022)$ & $220,552(344,991)$ & $102,287(96,175,108,687)$ & $<.001$ & $163,862(150,714,177,947)$ & $<.001$ \\
\hline
\end{tabular}

\section{Cl confidence interval, ICU intensive care unit}

${ }^{\mathrm{a}}$ Differences in means are from gamma regression model (link $=\log$ ). Statistical comparisons are comparing Cohort 3 (pneumonia due to $S$. aureus) with Cohort 1 (no pneumonia, reference group); ie, Difference $=$ mean (Cohort 3) - mean (Cohort 1)

${ }^{\mathrm{b}}$ Differences in means are from gamma regression model (link $\left.=\log \right)$. Statistical comparisons are comparing Cohort 4 (pneumonia due to $P$. aeruginosa) with Cohort 1 (no pneumonia, reference group); ie, Difference = mean (Cohort 4) - mean (Cohort 1)

costs include $\$ 0$ costs. All costs adjusted for calendar year, reported in 2012 dollars. All costs rounded to nearest dollar

All statistical models were controlled for the following variables: age (continuously), gender, health plan type, geographic region, and DCl comorbidity score. Models of index hospitalization costs and post-index costs All sta 1 so controled for were also contrition 12 mon [0 vs $1+]$, outpatient and office visits [continuous]), and prior antibiotic use during the 12 month pre-index period ( 0 vs. 1+) 
90 day) periods. Mean total costs for the index hospitalization were approximately 4 and 6 times greater in patients with $S$. aureus or $P$. aeruginosa pneumonia, respectively, than in those in the control group, resulting in incremental costs of $>\$ 100,000$.

During the first 30 days after index hospitalization discharge, patients in the $S$. aureus cohort incurred roughly $74 \%$ higher mean costs than the control cohort ( $\$ 8,243$ vs \$4732), and the $P$. aeruginosa cohort incurred more than double the mean cost compared with the control cohort. A similar pattern persisted during the 90 days following index hospitalization discharge.

During the index and 90-day post discharge periods inpatient costs are the major contributor for the overall costs in the $S$. aureus and $P$. aeruginosa cohorts and controls (>95\% vs $87.3 \%$, respectively). Inpatient and total costs were, respectively, $\$ 156,259$ and $\$ 164,437$ in the $S$. aureus pneumonia cohort and $\$ 226,631$ and $\$ 235,381$ in the $P$. aeruginosa pneumonia cohort compared with $\$ 38,765$ and $\$ 44,427$ for the control cohort (Table 1).

\section{Mortality within 90 days of discharge}

Patients with pneumonia due to $S$. aureus or P. aeruginosa experienced significantly $(\mathrm{P}<.001)$ higher rates of mortality during the index hospitalization and the 90-day follow up period versus controls (Table 2). The rate of all-cause mortality from the index hospitalization through the end of the 90-day follow up period was also significantly higher $(P<.001)$ in these cohorts. The mortality rates during the 90 days post-index period for patients who survived the index hospitalization were both significantly higher than the rate in controls $(P<.001$ for both). Overall survival rates during the post-index period (following index hospitalization discharge) were significantly lower $(P<.001)$ in the $S$. aureus and $P$. aeruginosa cohorts compared with the control group.

\section{Healthcare utilization}

Pre-index healthcare utilization in the $S$. aureus and $P$. aeruginosa cohorts was greater than in the control group. Patients who acquired these pneumonias during the index hospitalization had higher rates of inpatient hospitalizations and longer lengths of stay in the 12 months before the index hospitalization versus controls (Additional file 1: Table S3). The mean number of pre-index emergency room, office and outpatient visits was also generally higher among $S$. aureus and $P$. aeruginosa pneumonia patients than in controls (Additional file 1: Table S3). The percentage of patients with $\geq 1$ visit to a skilled nursing facility in the pre-index period was much higher in both the $S$. aureus and $P$. aeruginosa cohorts than in the control cohort $(P<.001)$. Antibiotic use was also significantly higher (Additional file 1: Table S3).

Healthcare utilization during the index hospitalization was higher among the $S$. aureus and P. aeruginosa cohorts

Table 2 All-cause mortality of ICU patients with S. aureus or P. aeruginosa pneumonia versus controls

\begin{tabular}{|c|c|c|c|c|c|c|c|}
\hline & \multirow{2}{*}{\multicolumn{3}{|c|}{ Cohorts of interest }} & \multicolumn{4}{|l|}{ Comparisons } \\
\hline & & & & \multicolumn{2}{|l|}{$\begin{array}{l}\text { S. aureus pneumonia } \\
\text { vs No pneumonia }\end{array}$} & \multicolumn{2}{|l|}{$\begin{array}{l}\text { P. aeruginosa pneumonia } \\
\text { vs No pneumonia }\end{array}$} \\
\hline & $\begin{array}{l}\text { No pneumonia } \\
(n=201,394)\end{array}$ & $\begin{array}{l}\text { S. aureus } \\
\text { pneumonia } \\
(n=2275)\end{array}$ & $\begin{array}{l}\text { P. aeruginosa } \\
\text { pneumonia } \\
(n=1007)\end{array}$ & $\begin{array}{l}\text { Odds Ratio/Difference }{ }^{a} \\
\text { (95\% Cl) }\end{array}$ & $P$ Value $^{a}$ & $\begin{array}{l}\text { Odds Ratio/Difference }{ }^{b} \\
\text { (95\% Cl) }\end{array}$ & $P$ Value \\
\hline \multicolumn{8}{|c|}{ Index hospitalization mortality } \\
\hline No. (\%) of patients & $6,188(3.1)$ & $363(16.0)$ & $203(20.2)$ & $5.99(5.34,6.72)$ & $<.001$ & $7.97(6.81,9.31)$ & $<.001$ \\
\hline \multicolumn{8}{|l|}{ Post-index mortality } \\
\hline No. (\%) of patients & $5,609(2.8)$ & $227(10.0)$ & 117 (11.6) & $3.87(3.37,4.45)$ & $<.001$ & $4.59(3.78,5.57)$ & $<.001$ \\
\hline Mean (SD) time to death ${ }^{c}$ & $32.8(27.1)$ & $25.5(25.8)$ & $28.1(28.4)$ & $-7.30(-10.51,-3.63)$ & $<.001$ & $-4.69(-9.44,1.02)$ & .10 \\
\hline Survival rate: & & & & $3.75(3.28,4.28)$ & $<.001$ & $4.40(3.66,5.28)$ & $<.001$ \\
\hline 30 days (\%) & 98.5 & 93.4 & 92.9 & & & & \\
\hline 90 days (\%) & 97.2 & 90.0 & 88.4 & & & & \\
\hline \multicolumn{8}{|l|}{ Index + post-index mortality } \\
\hline No. (\%) of patients & $11,797(5.9)$ & $590(25.9)$ & $320(31.8)$ & $5.63(5.11,6.19)$ & $<.001$ & $7.49(6.55,8.56)$ & $<.001$ \\
\hline Mean (SD) time to death ${ }^{c}$ & $24.9(28.3)$ & $46.4(47.6)$ & $63.5(59.3)$ & $21.53(17.39,26.07)$ & $<.001$ & $38.57(31.08,47.07)$ & $<.001$ \\
\hline
\end{tabular}

Cl confidence interval, ICU intensive care unit

${ }^{a}$ Odds ratio (OR) from Chi-square test is used for categorical variables, negative binomial models are used to test for differences between means for count variables (number of events and length of stay). Statistical comparisons are comparing Cohort 3 (pneumonia due to S. aureus) with Cohort 1 (no pneumonia, reference group); ie, Difference $=$ mean (Cohort 3) - mean (Cohort 1) and OR = Odds (Cohort 3)/Odds (Cohort 1)

${ }^{b}$ Odds ratio (OR) from Chi-square test is used for categorical variables, negative binomial models are used to test for differences between means for count variables (number of events and length of stay). Statistical comparisons are comparing Cohort 4 (pneumonia due to $P$. aeruginosa) with Cohort 1 (no pneumonia, reference group); i.e., Difference = mean (Cohort 4) - mean (Cohort 1) and OR= Odds (Cohort 4)/Odds (Cohort 1)

Including only patients with mortality 
versus the control cohort. Patients with $S$. aureus or $P$. aeruginosa pneumonia had significantly $(P<.001)$ longer overall mean hospital stays (37.9 and 55.4 days, respectively) versus controls (7.2 days), ICU stays (6.9 and 14.8 days, respectively vs 1.1 days), and higher rates of mechanical ventilation during the index hospitalization (Table 3).

During the 30 day post discharge period, a significantly $(P>.001)$ greater proportion of the survivors represented in the $S$. aureus and $P$. aeruginosa cohorts had inpatient hospitalizations and ICU visits) (Table 4) and skilled nursing facility visits (Additional file 1: Table S3) than did survivors in the control group, whereas the proportion of patients with office and outpatient visits were lower $(P<.001)$ in the $S$. aureus and $P$. aeruginosa cohorts versus the control group (Additional file 1: Table S3). A similar pattern in healthcare utilization was seen within 90 days after index discharge.

Survivors in the $S$. aureus and $P$. aeruginosa cohorts were more likely than controls to have a repeat pneumonia diagnosis during a subsequent hospital admission. Within the S. aureus cohort, during the 30-day and 90day follow up period, respectively, re-hospitalization that included a diagnosis of $S$. aureus pneumonia accounted for $9.6 \%$ and $10.0 \%$ of inpatient admissions and $21.4 \%$ and $15.3 \%$ of readmissions to the ICUs. For the $P$. aeruginosa cohort, re-hospitalization that included a diagnosis of $P$. aeruginosa pneumonia accounted for $16.7 \%$ and $20.0 \%$ of inpatient admissions, and $30.9 \%$ and $30.9 \%$ of ICU admissions during these periods (Table 4, Table 5). Most readmissions due to pneumonia during the follow up period occurred during the first 30 days, particularly for the patients with $S$. aureus pneumonia.

\section{Comorbidities}

The most common prespecified comorbidities identified by coding during the 12-month pre-index period across the 3 cohorts were hypertension, diabetes, coronary heart disease, anemia, and chronic obstructive pulmonary disease (Additional file 1: Table S4). Almost all comorbidities were more prevalent in patients with $S$. aureus or $P$. aeruginosa pneumonia than in controls. The mean DCI score 2.3 for the $S$. aureus and 2.5 for the $P$. aeruginosa cohort; both were significantly higher than in the control cohort (mean DCI = 1.7).

Additional file 1: Table S5 displays the prevalence of comorbidities within the cost quintiles for each cohort. Most of the comorbid conditions were not associated with significantly higher costs within the $S$. aureus and $P$. aeruginosa cohorts during the index hospitalization (ie, the odds ratio of the likelihood of appearing in a high cost quintile in the presence of the comorbidity versus the absence of the comorbidity was not significantly greater than 1; Table 4). Hepatitis B infection and solid organ transplant were associated with higher costs within the $S$. aureus cohort only, but the $P$ values were of marginal significance given the small sample sizes.

Nearly half of the comorbid conditions, including 4 cardiovascular conditions, were associated with significantly lower index hospitalization costs for either one or both of the $S$. aureus and P. aeruginosa cohorts (Table 6). Congestive heart failure, peripheral artery disease, and chronic obstructive pulmonary disease were associated with significantly lower costs in both the $S$. aureus and $P$. aeruginosa cohorts. Additionally, myocardial infarction, other coronary heart disease, and dementia were associated with lower costs in the S. aureus cohort; asthma, immunosuppression, human immunodeficiency

Table 3 Healthcare resource utilization for ICU patients with S. aureus or P. aeruginosa pneumonia versus controls during index hospitalization

\begin{tabular}{|c|c|c|c|c|c|c|c|}
\hline & \multicolumn{3}{|c|}{ Cohorts of interest } & \multicolumn{2}{|c|}{$\begin{array}{l}\text { S. aureus pneumonia vs No } \\
\text { pneumonia }\end{array}$} & \multicolumn{2}{|c|}{$\begin{array}{l}\text { P. aeruginosa pneumonia vs No } \\
\text { pneumonia }\end{array}$} \\
\hline & \multirow{3}{*}{$\begin{array}{l}\text { No } \\
\text { pneumonia } \\
N=201,394\end{array}$} & \multirow{3}{*}{$\begin{array}{l}\begin{array}{l}\text { S. aureus } \\
\text { pneumonia }\end{array} \\
\mathrm{N}=2275\end{array}$} & \multirow{3}{*}{$\begin{array}{l}P . \\
\text { aeruginosa } \\
\text { pneumonia } \\
N=1007\end{array}$} & \multirow{3}{*}{$\begin{array}{l}\text { Odds Ratio/Difference }{ }^{a} \\
(95 \% \mathrm{Cl})\end{array}$} & \multirow[t]{3}{*}{$P$ Value $^{a}$} & \multirow{3}{*}{$\begin{array}{l}\text { Odds Ratio/Difference }{ }^{\mathrm{b}} \\
(95 \% \mathrm{Cl})\end{array}$} & \multirow[t]{3}{*}{$P$ Value } \\
\hline & & & & & & & \\
\hline & & & & & & & \\
\hline $\begin{array}{l}\text { Mean (SD) length of hospital } \\
\text { stay per patient }\end{array}$ & $7.2(9.6)$ & $37.9(39.4)$ & $55.4(54.9)$ & $30.69(29.41,32.00)$ & $<.001$ & $48.18(45.43,51.08)$ & $<.001$ \\
\hline $\begin{array}{l}\text { Mean (SD) length of ICU/CCU } \\
\text { stay per patient }\end{array}$ & $1.1(2.1)$ & $6.9(21.1)$ & $14.8(36.6)$ & $5.75(5.59,5.91)$ & $<.001$ & $13.63(13.21,14.06)$ & $<.001$ \\
\hline $\begin{array}{l}\text { No. (\%) of patients with } \\
\text { mechanical ventilation }\end{array}$ & $14,960(7.4)$ & $1,425(62.6)$ & $627(62.3)$ & $20.89(19.16,22.78)$ & $<.001$ & $20.56(18.08,23.38)$ & $<.001$ \\
\hline
\end{tabular}

Cl confidence interval, ICU intensive care unit

${ }^{a}$ Odds ratio (OR) from Chi-square test is used for categorical variables, negative binomial models are used to test for differences between means for count variables (number of events and length of stay). Statistical comparisons are comparing Cohort 3 (pneumonia due to S. aureus) with Cohort 1 (no pneumonia, reference group); ie, Difference $=$ mean (Cohort 3) - mean (Cohort 1 ) and OR = Odds (Cohort 3)/Odds (Cohort 1)

${ }^{b}$ Odds ratio (OR) from Chi-square test is used for categorical variables, negative binomial models are used to test for differences between means for count variables (number of events and length of stay). Statistical comparisons are comparing Cohort 4 (pneumonia due to $P$. aeruginosa) to Cohort 1 (no pneumonia, reference group); ie, Difference $=$ mean $($ Cohort 4$)-$ mean $($ Cohort 1$)$ and OR= Odds (Cohort 4)/Odds (Cohort 1) 
Table 4 Healthcare resource utilization for ICU patients with S. aureus or P. aeruginosa pneumonia versus controls through 30 days post index hospitalization

\begin{tabular}{|c|c|c|c|c|c|c|c|}
\hline & \multicolumn{3}{|c|}{ Cohorts of interest } & \multicolumn{2}{|c|}{$\begin{array}{l}\text { S. aureus pneumonia vs No } \\
\text { pneumonia }\end{array}$} & \multicolumn{2}{|c|}{$\begin{array}{l}\text { P. aeruginosa pneumonia vs No } \\
\text { pneumonia }\end{array}$} \\
\hline & $\begin{array}{l}\text { No } \\
\text { pneumonia }\end{array}$ & $\begin{array}{l}\text { S. aureus } \\
\text { pneumonia }\end{array}$ & $\begin{array}{l}\text { P. aeruginosa } \\
\text { pneumonia }\end{array}$ & $\begin{array}{l}\text { Odds Ratio/Difference } \\
(95 \% \mathrm{Cl})\end{array}$ & $P$ Value $^{\mathrm{a}}$ & $\begin{array}{l}\text { Odds Ratio/Difference }{ }^{b} \\
(95 \% \mathrm{Cl})\end{array}$ & $\begin{array}{l}P \\
\text { Value }^{b}\end{array}$ \\
\hline No. of patients completing 30 day follow up period ${ }^{c}$ & $n=192,204$ & $n=1762$ & $n=732$ & & & & \\
\hline \multicolumn{8}{|l|}{ All-cause inpatient hospitalizations } \\
\hline No. (\%) of patients with $\geq 1$ event & $29,451(15.3)$ & $480(27.2)$ & $228(31.1)$ & $2.07(1.86,2.30)$ & $<.001$ & $2.50(2.14,2.93)$ & $<.001$ \\
\hline Mean (SD) events per patient with at least 1 event & $1.3(0.7)$ & $1.6(1.3)$ & $1.5(1.2)$ & $0.30(0.19,0.42)$ & $<.001$ & $0.24(0.09,0.41)$ & .002 \\
\hline Mean (SD) length of stay per patient with at least 1 event & $5.4(5.5)$ & $8.4(7.3)$ & $9.5(7.7)$ & $3.02(2.38,3.71)$ & $<.001$ & $4.15(3.13,5.29)$ & $<.001$ \\
\hline No. (\%) of patients with $\geq 1$ hospitalization for pneumonia due to $S$. aureus & $52(0.03)$ & $46(2.6)$ & $13(1.8)$ & $99.06(66.43,147.71)$ & $<.001$ & $66.81(36.22,123.23)$ & $<.001$ \\
\hline No. (\%) of patients with $\geq 1$ hospitalization for pneumonia due to $P$. aeruginosa & $31(0.02)$ & $16(0.9)$ & $38(5.2)$ & $56.81(31.02,104.05)$ & $<.001$ & $339.43(210.00,548.64)$ & $<.001$ \\
\hline \multicolumn{8}{|l|}{ ICU stays } \\
\hline No. (\%) of patients with $\geq 1$ event & $5027(2.6)$ & $84(4.8)$ & $55(7.5)$ & $1.86(1.50,2.33)$ & $<.001$ & $3.03(2.30,3.99)$ & $<.001$ \\
\hline Mean (SD) events per patient with at least 1 event & $1.0(0.2)$ & $1.0(0.1)$ & $1.0(0.0)$ & $-0.01(-0.21,0.23)$ & .89 & $-0.03(-0.26,0.28)$ & .85 \\
\hline Mean (SD) length of stay per patient with at least 1 event & $1.3(1.6)$ & $1.4(3.0)$ & $1.3(1.2)$ & $0.10(-0.14,0.40)$ & .44 & $0.02(-0.27,0.38)$ & .91 \\
\hline No. (\%) of patients with $\geq 1$ hospitalization for pneumonia due to $S$. aureus & $34(0.02)$ & $18(1.0)$ & $7(1.0)$ & $58.34(32.88,103.49)$ & $<.001$ & $54.57(24.11,123.50)$ & $<.001$ \\
\hline No. (\%) of patients with $\geq 1$ hospitalization for pneumonia due to $P$. aeruginosa & $18(0.01)$ & $8(0.5)$ & $17(2.3)$ & $48.72(21.16,112.18)$ & $<.001$ & $253.83(130.29,494.51)$ & $<.001$ \\
\hline
\end{tabular}

\section{Cl confidence interval, ICU intensive care unit}

${ }^{a}$ Odds ratio (OR) from Chi-square test is used for categorical variables, negative binomial models are used to test for differences between means for count variables (number of events and length of stay). Statistical

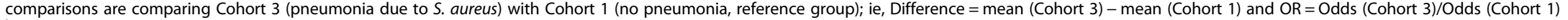

bodds ratio (OR) from Chi-square test is used for categorical variables, negative binomial models are used to test for differences between means for count variables (number of events and length of stay). Statistical comparisons are comparing Cohort 4 (pneumonia due to $P$. aeruginosa) to Cohort 1 (no pneumonia, reference group); ie, Difference $=$ mean $($ Cohort 4$)-$ mean $($ Cohort 1$)$ and OR $=$ Odds $($ Cohort 4)/Odds $($ Cohort 1$)$ CPost-index utilization results include only patients who survived to the end of the 30 or 90 day post-discharge period 
Table 5 Healthcare resource utilization for ICU patients with S. aureus or P. aeruginosa pneumonia versus controls through 90 days post index hospitalization

\begin{tabular}{|c|c|c|c|c|c|c|c|}
\hline & \multicolumn{3}{|c|}{ Cohorts of interest } & \multicolumn{2}{|c|}{$\begin{array}{l}\text { S. aureus pneumonia vs No } \\
\text { pneumonia }\end{array}$} & \multicolumn{2}{|c|}{$\begin{array}{l}\text { P. aeruginosa pneumonia vs No } \\
\text { pneumonia }\end{array}$} \\
\hline & $\begin{array}{l}\text { No } \\
\text { pneumonia }\end{array}$ & $\begin{array}{l}\text { S. aureus } \\
\text { pneumonia }\end{array}$ & $\begin{array}{l}P . \\
\text { aeruginosa } \\
\text { pneumonia }\end{array}$ & $\begin{array}{l}\text { Odds Ratio/Difference } \\
\text { (95\% Cl) }\end{array}$ & $P$ Value ${ }^{\mathrm{a}}$ & $\begin{array}{l}\text { Odds Ratio/Difference }{ }^{b} \\
(95 \% \text { Cl) }\end{array}$ & $P$ Value $^{\mathrm{b}}$ \\
\hline $\begin{array}{l}\text { No. of patients completing } 90 \text { day } \\
\text { follow up period }\end{array}$ & $n=189,597$ & $n=1685$ & $n=687$ & & & & \\
\hline \multicolumn{8}{|l|}{ All-cause inpatient hospitalizations } \\
\hline No. (\%) of patients with $\geq 1$ event & $44,246(23.3)$ & $641(38.0)$ & $295(42.9)$ & $2.02(1.83,2.23)$ & $<.001$ & $2.48(2.13,2.88)$ & $<.001$ \\
\hline $\begin{array}{l}\text { Mean (SD) events per patient with } \\
\text { at least } 1 \text { event }\end{array}$ & $1.5(1.0)$ & $1.9(2.0)$ & $2.0(1.9)$ & $0.45(0.34,0.56)$ & $<.001$ & $0.46(0.31,0.63)$ & $<.001$ \\
\hline $\begin{array}{l}\text { Mean (SD) length of stay per patient } \\
\text { with at least } 1 \text { event }\end{array}$ & $7.8(10.7)$ & $14.7(18.4)$ & $17.5(19.8)$ & $6.96(5.82,8.19)$ & $<.001$ & $9.79(7.85 .11 .97)$ & $<.001$ \\
\hline $\begin{array}{l}\text { No. (\%) of patients with } \geq 1 \\
\text { hospitalization for pneumonia } \\
\text { due to } S \text {. aureus }\end{array}$ & $112(0.06)$ & $64(3.8)$ & $20(2.9)$ & $66.80(48.95,91.17)$ & $<.001$ & $50.74(31.34,82.14)$ & $<.001$ \\
\hline $\begin{array}{l}\text { No. }(\%) \text { of patients with } \geq 1 \\
\text { hospitalization for pneumonia } \\
\text { due to } P \text {. aeruginosa }\end{array}$ & $63(0.03)$ & $29(1.7)$ & $59(8.6)$ & $52.69(33.85,82.01)$ & $<.001$ & $282.61(196.46,406.55)$ & $<.001$ \\
\hline \multicolumn{8}{|l|}{ ICU stays } \\
\hline No. (\%) of patients with $\geq 1$ event & $9,399(5.0)$ & $163(9.7)$ & $97(14.1)$ & $2.05(1.75,2.42)$ & $<.001$ & $3.15(2.54,3.91)$ & $<.001$ \\
\hline $\begin{array}{l}\text { Mean (SD) events per patients with } \\
\text { at least } 1 \text { event }\end{array}$ & $1.1(0.4)$ & $1.1(0.3)$ & $1.2(0.5)$ & $-0.02(-0.17,0.15)$ & .80 & $0.10(-0.10,0.34)$ & .37 \\
\hline $\begin{array}{l}\text { Mean (SD) length of stay per patients } \\
\text { with at least } 1 \text { event }\end{array}$ & $1.6(3.6)$ & $3.5(10.1)$ & $1.9(3.6)$ & $1.92(1.46,2.44)$ & $<.001$ & $0.34(-0.01,0.77)$ & .06 \\
\hline $\begin{array}{l}\text { No. (\%) of patients with } \geq 1 \\
\text { hospitalization for pneumonia } \\
\text { due to } S \text {. aureus }\end{array}$ & $66(0.03)$ & $25(1.5)$ & $9(1.3)$ & $43.25(27.23,68.70)$ & $<.001$ & $38.13(18.93,76.81)$ & $<.001$ \\
\hline $\begin{array}{l}\text { No. (\%) of patients with } \geq 1 \\
\text { hospitalization for pneumonia } \\
\text { due to } P \text {. aeruginosa }\end{array}$ & $32(0.02)$ & $17(1.0)$ & $30(4.4)$ & $60.38(33.46,108.94)$ & $<.001$ & $270.47(163.40,447.69)$ & $<.001$ \\
\hline
\end{tabular}

Cl confidence interval, ICU intensive care unit

${ }^{a}$ Odds ratio (OR) from Chi-square test is used for categorical variables, negative binomial models are used to test for differences between means for count variables (number of events and length of stay). Statistical comparisons are comparing Cohort 3 (pneumonia due to S. aureus) with Cohort 1 (no pneumonia, reference group); ie, Difference = mean (Cohort 3) - mean (Cohort 1) and OR= Odds (Cohort 3)/Odds (Cohort 1)

${ }^{b}$ Odds ratio (OR) from Chi-square test is used for categorical variables, negative binomial models are used to test for differences between means for count variables (number of events and length of stay). Statistical comparisons are comparing Cohort 4 (pneumonia due to $P$. aeruginosa) to Cohort 1 (no pneumonia, reference group); ie, Difference $=$ mean (Cohort 4$)-$ mean (Cohort 1$)$ and OR= Odds (Cohort 4)/Odds (Cohort 1)

'Post-index utilization results include only patients who survived to the end of the 30 or 90 day post-discharge period

virus infection, and Hepatitis B infection were associated with lower costs in the $P$. aeruginosa cohort. However, the results for immunosuppression, human immunodeficiency virus, and hepatitis B should be interpreted with caution due to the small sample sizes (Additional file 1: Table S4).

An exploratory analysis of congestive heart failure was performed that stratified patients by age and mortality (Additional file 1: Table S6) to control for potential correlations between comorbidities and patient characteristics and outcomes (Medicare eligibility and death) that may result in lower hospitalization costs. The results showed odds ratios $<1$ despite these additional adjustments in the models. Similar results were found in exploratory analyses of other pre-specified conditions with odds ratios $<1$.

\section{Discussion}

This is the largest US claims database study of healthcare costs and outcomes for ICU patients with a diagnosis of $S$. aureus or $P$. aeruginosa pneumonia. Our findings highlight the comprehensive economic consequences attributed to S. aureus and P. aeruginosa pneumonia and can permit policy makers, payers, and healthcare providers to assess the effect of prevention or therapeutic efforts on the cost and morbidity of these ICU infections.

In our study, ICU patients with pneumonia had substantially higher healthcare costs during the index admission: $>\$ 213,000$ for $P$. aeruginosa pneumonia and $>\$ 146,000$ for with $S$. aureus pneumonia versus $>\$ 33,000$ for patients without pneumonia. Increased utilization continued after index hospitalization discharge, 
Table 6 Impact of comorbidities on index hospitalization costs for ICU patients with S. aureus or P. aeruginosa pneumonia

\begin{tabular}{|c|c|c|c|c|}
\hline \multirow[t]{3}{*}{ Comorbidity } & \multicolumn{2}{|c|}{ S. aureus pneumonia } & \multicolumn{2}{|c|}{ P. aeruginosa pneumonia } \\
\hline & Odds Ratio $^{a}$ & $P$ Value $^{a}$ & Odds Ratio ${ }^{a}$ & $P$ Value $^{\mathrm{a}}$ \\
\hline & \multicolumn{2}{|l|}{$(95 \% \mathrm{Cl})$} & \multicolumn{2}{|l|}{$(95 \% \mathrm{Cl})$} \\
\hline Diabetes & $1.10(0.93,1.31)$ & .27 & $1.12(0.86,1.46)$ & .40 \\
\hline Myocardial infarction & $0.58(0.42,0.82)$ & .002 & $1.14(0.71,1.82)$ & .59 \\
\hline Congestive heart failure & $0.74(0.60,0.92)$ & .007 & $0.72(0.52,0.99)$ & .04 \\
\hline Peripheral artery disease & $0.69(0.54,0.88)$ & .003 & $0.70(0.49,0.99)$ & .04 \\
\hline Stroke, TIA, cerebrovascular disease & $0.81(0.65,1.01)$ & .06 & $1.17(0.83,1.65)$ & .37 \\
\hline Hypertension & $0.96(0.80,1.14)$ & .62 & $1.16(0.88,1.52)$ & .29 \\
\hline Other coronary heart disease & $0.80(0.66,0.97)$ & .03 & $0.94(0.71,1.24)$ & .65 \\
\hline Anemia & $0.88(0.72,1.07)$ & .20 & $1.12(0.84,1.50)$ & .44 \\
\hline COPD & $0.68(0.57,0.82)$ & $<.001$ & $0.46(0.35,0.60)$ & $<.001$ \\
\hline Asthma & $0.95(0.76,1.20)$ & .67 & $0.60(0.43,0.84)$ & .003 \\
\hline Renal disease & $0.90(0.70,1.15)$ & .38 & $1.06(0.73,1.52)$ & .77 \\
\hline Chronic liver disease & $1.17(0.87,1.58)$ & .29 & $0.85(0.54,1.33)$ & .47 \\
\hline Neutropenia & $1.53(0.91,2.58)$ & .11 & $0.59(0.29,1.19)$ & .14 \\
\hline Immunosuppression & $1.05(0.51,2.16)$ & .89 & $0.39(0.16,0.96)$ & .04 \\
\hline HIV & $1.00(0.34,2.90)$ & .996 & $0.27(0.08,0.88)$ & .03 \\
\hline Hepatitis B & $4.77(1.05,21.72)$ & .04 & $0.04(0.00,0.74)$ & .03 \\
\hline Hepatitis C & $0.96(0.45,2.05)$ & .92 & $0.57(0.19,1.70)$ & .31 \\
\hline Obesity/Overweight & $1.03(0.78,1.36)$ & .86 & $1.15(0.76,1.75)$ & .50 \\
\hline Dementia & $0.55(0.35,0.86)$ & .009 & $1.76(0.75,4.15)$ & .20 \\
\hline Dialysis & $0.62(0.33,1.15)$ & .13 & $1.21(0.53,2.78)$ & .65 \\
\hline Leukemia & $0.61(0.27,1.35)$ & .22 & $1.03(0.43,2.47)$ & .95 \\
\hline Lymphoma & $1.60(0.91,2.82)$ & .11 & $1.21(0.56,2.58)$ & .63 \\
\hline Cancer other than leukemia or lymphoma & $0.88(0.58,1.34)$ & .56 & $0.93(0.51,1.70)$ & .82 \\
\hline Bone marrow transplant & $0.89(0.15,5.30)$ & .89 & $0.61(0.11,3.43)$ & .58 \\
\hline Solid organ transplant & $1.90(1.02,3.53)$ & .04 & $1.31(0.66,2.57)$ & .44 \\
\hline
\end{tabular}

$\mathrm{Cl}$ confidence interval, COPD chronic obstructive pulmonary disease, HIV human immunodeficiency virus, ICU intensive care unit, TIA transient ischemic attack ${ }^{a}$ Ordinal logistic regression odds ratio (OR) is used to test the association between the presence of each comorbidity and index hospitalization cost quintiles, ie, $\mathrm{OR}=$ Odds (patient with comorbidity in high cost quintile)/ Odds (patient without comorbidity in high cost quintile). OR $>1$ means presence of the comorbidity leads to greater costs, whereas $\mathrm{OR}<1$ means presence of the comorbidity leads to lower costs

All statistical models were controlled for the following variables: age (continuously), gender, health plan type, geographic region, prior inpatient hospitalization costs during the 12 month pre-index period, prior healthcare utilization during the 12 month pre-index period (inpatient stays [0 vs $1+]$, emergency room visits [0 vs $1+]$, outpatient and office visits [continuous]), and prior antibiotic use during the 12 month pre-index period $(0$ vs. $1+)$

with total healthcare costs through 90 days post discharge of $>\$ 17,000$ for patients with $S$. aureus pneumonia and > $\$ 22,000$ for patients with $P$. aeruginosa pneumonia versus $>\$ 10,000$ for patients without pneumonia. Patients with $S$. aureus or $P$. aeruginosa pneumonia had estimated incremental index hospitalization costs of $\$ 100,000$ $\$ 160,000$ and total healthcare costs of $\$ 107,000-\$ 167,000$ versus ICU patients without pneumonia. In previous studies of the general US inpatient population, the mean cost of hospital care in patients with hospital-acquired pneumonia was $\$ 72,000$ versus $\$ 46,400$ to $\$ 65,292$ for patients without pneumonia [2, 22]. A German study reported an excess mean cost of DM29,610 (equivalent to $\$ 16,824$ in 2001) for hospital-acquired pneumonia in ICU patients compared with ICU patients without pneumonia [23]. Previous US studies in VAP patients estimated that the incremental costs were $\$ 39,000$ to $\$ 100,000$ for VAP patients versus ICU patients without VAP, which is consistent with our observations [24, 25].

Our findings showed that $S$. aureus pneumonia and, especially, $P$. aeruginosa pneumonia had prolonged hospitalizations ( $>48$ days longer for $P$. aeruginosa and $>30$ days longer for S. aureus) and ICU stays (approximately 15 days for those with $P$. aeruginosa pneumonia, 7 days for those with $S$. aureus pneumonia, compared with 1 day for those without pneumonia). Mortality was also substantially higher: $>20 \%$ of those with $P$. aeruginosa pneumonia and $16 \%$ of those with $S$. aureus pneumonia died during the 
index hospitalization versus approximately $3 \%$ of control patients, and this trend continued after discharge. The findings of longer ICU lengths of stay and longer hospital lengths of stay are consistent with prior studies [2, 22-24].

We found significantly higher rehospitalization rates in patients with $S$. aureus and $P$. aeruginosa pneumonia versus controls. Readmission after discharge can be costly and problematic for the patient, hospitals, and payers. It was estimated that hospital readmissions cost Medicare $\$ 17.5$ billion alone annually in the United States [26]. Financial penalties are now incurred for hospitals with excess 30-day readmission in the United States. Therefore, preventing $S$. aureus and $P$. aeruginosa pneumonias could reduce the substantial costs of and healthcare utilization associated with rehospitalization in patients with these infections, resulting in benefits for patients, payers, and healthcare providers.

ICU patients with $S$. aureus or $P$. aeruginosa pneumonia were older, more likely to be male, and in poorer general health than those who did not have pneumonia. Most patients ( $>62 \%)$ were mechanically ventilated; this is likely to be an underestimate, because mechanical ventilation is not uniformly recorded in a claims database. These mechanically-ventilated patients represent both those with VAP and those with non-ventilatorassociated pneumonia that required ventilator support. The demographic skew to older, male patients has been previously reported in patients with VAP; however, in some studies, patients with VAP were younger than were those without VAP, which may reflect the contribution of trauma patients to the demographics [24, 27].

The findings regarding the impact of comorbid conditions on the hospitalization costs of S. aureus or P. aeruginosa pneumonia are unique to the present study. The results indicate that while the presence of comorbid conditions increases the likelihood that an ICU patient will develop $S$. aureus or $P$. aeruginosa pneumonia, the presence of comorbidities does not inflate the costs of hospital care for the patient. A number of comorbid conditions were associated with lower, rather than higher, hospitalization costs for S. aureus or P. aeruginosa pneumonia. Exploratory analysis demonstrated that the lower costs are not solely due to higher mortality or higher rates of Medicare coverage among patients with the comorbid conditions. It is unknown why these inverse associations exist.

Claims data represent an excellent starting point for the examination of health outcomes, treatment patterns, healthcare resource utilization, and costs. However, our analyses have several limitations. This study was retrospective and used ICD-9 codes to include and exclude subjects from specific cohorts, a method associated with known limitations [28]. Because the included codes were selected to be specific rather than sensitive, the numbers of patients identified cannot be used to establish incidence rates. It is possible that other pneumonia codes, including the code for VAP, included some episodes of $P$. aeruginosa or $S$. aureus pneumonia. Comorbidities were identified by their presence in coded diagnoses in the 12 months before the index hospitalization; this approach is likely very sensitive and intended to capture as many comorbid conditions as possible but likely has limited specificity due to the criteria of only requiring one diagnosis code. Diagnoses made during the index hospitalization were not included, because the goal of the current analysis was to assess the information available to clinicians upon admission; this may have contributed to underdiagnosis of co-morbid conditions. Future studies may want to examine the association between costs and both pre-existing and new comorbidities to explore the impact of how comorbidities are defined. Additionally, separating patients into those hospitalized for a lifethreatening pneumonia from those who acquired pneumonia in the hospital would be useful in assessing prevention strategies, however claims database analyses do not permit separation into these groups. Furthermore, the database only included patients who had commercial healthcare insurance and may not be representative of the overall US population; patients covered solely by Medicare or Medicaid were excluded. However, the claims database comprised patients aged $\geq 18$ years in a managed care setting and included substantial numbers of subjects of Medicare age; moreover, results may be generalizable to similar patient groups. Despite these limitations, our results provide accurate assessments of resource utilizations and costs in these patients due to appropriate adjustments in the statistical models.

\section{Conclusion}

Overall, these results indicate that $S$. aureus and P. aeruginosa pneumonia in ICU patients impose substantial costs, healthcare utilization, and burden for patients [29]. The demand for critical care services is increasing as the aging US population requires a greater level of critical and end of life care, and emergency departments are accelerating their rates of ICU admissions [30]. These services are costly; in 2005, ICUs in the United States accounted for 23.2 million patient days and $\$ 81.7$ billion in expenditures. Our results highlight the economic importance of effective interventions to reduce the burden of $S$. aureus and $P$. aeruginosa pneumonia in ICUs in the United States.

\section{Additional file}

Additional file 1: Table S1. Baseline demographic characteristics of ICU patients with $S$. aureus or $P$. aeruginosa pneumonia versus controls. Table S2. Non-hospital medical and pharmacy costs for ICU patients 
with $S$. aureus or $P$. aeruginosa pneumonia versus controls. Table S3. Pre- and post-index healthcare resource utilization for ICU patients with $S$. aureus or $P$. aeruginosa pneumonia versus controls. Table S4. Comorbidities of ICU patients with S. aureus and P. aeruginosa pneumonia versus controls. Table S5. Prevalence of comorbid conditions by quintile of index hospitalization cost for ICU patients with S. aureus or P. aeruginosa pneumonia versus controls. Table S6. Exploratory analysis of the impact of congestive heart failure on index hospitalization costs in ICU patients with hospital acquired pneumonia due to S. aureus. Table S7. Healthcare resource utilization for ICU patients with $S$. aureus or $P$. aeruginosa pneumonia versus controls pre-index hospitalization (12 months).

\section{Competing interests}

MHK, JF, HSJ are current or former employees of Medlmmune and shareholders or holders of unvested shares. DMK, SZ, and OT are employees of HealthCore, Inc.

\section{Authors' contributions}

HSJ, MHK, DMK, OT, and JF were involved in the study, concept, and design. DMK, OT, and SZ were responsible for data acquisition. All authors contributed to data analysis and manuscript writing. Assistance with formatting of the manuscript was provided by John E. Fincke, PhD, and Anny Wu, PharmD, of Complete Healthcare Communications, Inc., (Chadds Ford, PA, USA) and funded by Medlmmune. All authors read and approved the final manuscript.

\section{Acknowledgement}

We would like to thank Kellie Ryan, MPH, Lindsey Trickett, MBA, Michael McCarthy, MD, Frank Sifakis, PhD, and Kathryn Jensen, MSc, for helpful discussions during the early stage of study concept and design. We gratefully acknoweldege Kellie Ryan for her help in formatting of manuscript and coordinating the manuscript resubmission.

\section{Financial support}

The study was funded by Medlmmune.

\section{Author details}

${ }^{1}$ Medlmmune, Gaithersburg, USA. ${ }^{2}$ Current author address: Sanofi Pasteur, Swiftwater, PA, USA. ${ }^{3}$ Healthcore, Wilmington, DE, USA.

\section{Received: 24 November 2014 Accepted: 9 June 2015}

\section{Published online: 21 June 2015}

\section{References}

1. Gaynes R, Edwards JR. National Nosocomial Infections Surveillance System. Overview of nosocomial infections caused by gram-negative bacilli. Clin Infect Dis. 2005;41:848-54.

2. Kollef MH, Shorr A, Tabak YP, Gupta V, Liu LZ, Johannes RS. Epidemiology and outcomes of health-care-associated pneumonia: Results from a large US database of culture-positive pneumonia. Chest. 2005;128:3854-62.

3. Hoffken G, Niederman MS. Nosocomial pneumonia: the importance of a de-escalating strategy for antibiotic treatment of pneumonia in the ICU. Chest. 2002;122:2183-96.

4. Esperatti M, Ferrer M, Theessen A, Liapikou A, Valencia M, Saucedo LM, et al. Nosocomial pneumonia in the intensive care unit acquired by mechanically ventilated versus nonventilated patients. Am J Respir Crit Care Med. 2010;182:1533-9.

5. Park DR. The microbiology of ventilator-assisted pneumonia. Resp Care. 2005:50:742-65.

6. Shorr AF, Tabak YP, Gupta V, Johannes RS, Liu LZ, Kollef MH. Morbidity and cost burden of methicillin-resistant Staphylococcus aureus in early onset ventilator-associated pneumonia. Crit Care. 2006;10:R97.

7. Bou R, Lorente L, Aguilar A, Perpiñán J, Ramos P, Peris M, et al. Hospital economic impact of an outbreak of Pseudomonas aeruginosa infections. J Hosp Infect. 2009;71:138-42.

8. Noskin GA, Rubin RJ, Schentag JJ, Kluytmans J, Hedblom EC, Smulders M, et al. The burden of Staphylococcus aureus infections on hospitals in the United States: an analysis of the 2000 and 2001 Nationwide Inpatient Sample Database. Arch Intern Med. 2005;165:1756-61.
9. Rello J, Diaz E. Pneumonia in the intensive care unit. Crit Care Med. 2003;31:2544-51.

10. Rubin RJ, Harrington CA, Poon A, Dietrich K, Greene JA, Moiduddin A. The economic impact of Staphylococcus aureus infection in New York City hospitals. Emerg Infect Dis. 1999;5:9-17.

11. Schreiber MP, Chan CM, Shorr AF. Bacteremia in Staphylococcus aureus pneumonia: outcomes and epidemiology. J Crit Care. 2011;26:395-401.

12. Shorr A, Lodise TP. Burden of Methicillin-resistant Staphylococcus aureus on healthcare cost and resource utilization. ISMR Update. 2006;1:1-12.

13. Shorr AF, Combes A, Kollef MH, Chastre J. Methicillin-resistant Staphylococcus aureus prolongs intensive care unit stay in ventilator-associated pneumonia, despite initially appropriate antibiotic therapy. Crit Care Med. 2006;34:700-6.

14. Centers for Disease Control and Prevention. Antibiotic Resistance Threats in the United States, 2013. Centers for Disease Control and Prevention; 2013. http://www.cdc.gov/drugresistance/pdf/ar-threats-2013-508.pdf

15. Carmeli Y, Troillet N, Karchmer AW, Samore MH. Health and economic outcomes of antibiotic resistance in Pseudomonas aeruginosa. Arch Intern Med. 1999;159:1127-32.

16. Lautenbach E, Synnestvedt M, Weiner MG, Bilker WB, Vo L, Schein J, et al. Imipenem resistance in Pseudomonas aeruginosa: emergence, epidemiology, and impact on clinical and economic outcomes. Infect Control Hosp Epidemiol. 2010;31:47-53.

17. Mauldin PD, Salgado CD, Hansen IS, Durup DT, Bosso JA. Attributable hospital cost and length of stay associated with health care-associated infections caused by antibiotic-resistant gram-negative bacteria. Antimicrob Agents Chemother. 2010;54:109-15.

18. Morales E, Cots F, Sala M, Comas M, Belvis F, Riu M, et al. Hospital costs of nosocomial multi-drug resistant Pseudomonas aeruginosa acquisition. BMC Health Serv Res. 2012;12:122

19. DiGiandomenico A, Camara M, Keller A, Thaden JT, Guenther C, Shire N, Fowler VG, Stover CK. MEDI3902 PcrV and Psl Target Validation in Pseudomonas aeruginosa Clinical Isolates and Analysis of Target Activity in Matched Patient Serum Samples. In: Interscience Conference on Antimicrobial Agents and Chemotherapy 2014 Online Abstracts. American Society for Microbiology. 2014. http://www.icaaconline.com/php/ icaac2014abstracts/data/index.htm.

20. Oganesyan V, Peng L, Damschroder MM, Cheng L, Sadowska A, Tkaczyk C, et al. Mechanisms of neutralization of a human anti-a-toxin antibody. J Biol Chem. 2014:43:29874-80.

21. Nadathur SG. Comorbidity indexes from administrative datasets: what is measured? Aust Health Rev. 2011;35:507-11.

22. Eber MR, Laxminarayan R, Perencevich EN, Malani A. Clinical and economic outcomes attributable to health care-associated sepsis and pneumonia. Arch Intern Med. 2010;170:347-53.

23. Dietrich ES, Demmler M, Schulgen G, Fekec K, Mast O, Pelz K, et al. Nosocomial pneumonia: a cost-of-illness analysis. Infection. 2002;30:61-7.

24. Kollef MH, Hamilton CW, Ernst FR. Economic impact of ventilator-associated pneumonia in a large matched cohort. Infect Control Hosp Epidemiol. 2012;33:250-6

25. Restrepo Ml, Anzueto A, Arroliga AC, Afessa B, Atkinson MJ, Ho NJ, et al. Economic burden of ventilator-associated pneumonia based on total resource utilization. Infect Control Hosp Epidemiol. 2010;31:509-15.

26. Jencks SF, Williams MV, Coleman EA. Rehospitalizations among patients in the Medicare fee-for-service program. N Engl J Med. 2009;360:1418-28.

27. Rello J, Ollendorf DA, Oster G, Vera-Llonch M, Bellm L, Redman R, et al. VAP Outcomes Scientific Advisory Group. Epidemiology and outcomes of ventilator-associated pneumonia in a large US database. Chest. 2002;122:2115-21.

28. O'Malley KJ, Cook KF, Price MD, Wildes KR, Hurdle JF, Ashton CM. Measuring diagnoses: ICD code accuracy. Health Serv Res. 2005;40:1620-39.

29. Halpern NA, Pastores SM. Critical care medicine in the United States 2000-2005: An analysis of bed numbers, occupancy rates, payer mix, and costs. Crit Care Med. 2010;38:65-71.

30. Mullins PM, Goyal M, Pines JM. National growth in intensive care unit admissions from emergency departments in the United States from 2002 to 2009. Acad Emerg Med. 2013;20:479-86. 